\title{
Acúmulo de nutrientes em gérbera de vaso em função de fontes e doses de potássio
}

\author{
Amaralina C Guerrero; Dirceu M Fernandes; Fernanda Ludwig \\ UNESP-FCA, Dep ${ }^{\text {to }}$ Recursos Naturais, Ciência do Solo, C. Postal 237, 18610-907 Botucatu-SP; amaralina@fca.unesp.br; \\ dmfernandes@fca.unesp.br; ludwig.fernanda@yahoo.com.br
}

\section{RESUMO}

Entre as várias espécies ornamentais cultivadas de importância econômica encontra-se a gérbera, uma planta com boa durabilidade, resistência ao transporte e muito apreciada pelos consumidores. Apesar da expansão na produção comercial de plantas ornamentais, a pesquisa não tem acompanhado esse ritmo de crescimento, principalmente em relação à nutrição e a adubação. Objetivou-se com esse trabalho avaliar o teor e acúmulo de macronutrientes em plantas de gérbera em dois estádios da cultura (período vegetativo e reprodutivo), com o uso de diferentes concentrações e fontes de potássio. O experimento foi conduzido em casa de vegetação da UNESP em Botucatu (SP). O delineamento experimental adotado foi em blocos casualizados, empregando o esquema fatorial $2 \times 5$, duas fontes de potássio (silicato e cloreto) com cinco concentrações $(0$, $50,100,150$ e $200 \mathrm{mg} \mathrm{L}^{-1}$ de potássio, via solução nutritiva), quatro repetições e cinco plantas por parcela. Foi utilizada a cultivar Red, com centro escuro, para cultivo em vaso. Avaliaram-se os teores de macronutrientes ( $\mathrm{N}, \mathrm{P}, \mathrm{K}, \mathrm{Ca}, \mathrm{Mg}$ e S) nas folhas durante o período vegetativo e nas folhas e flores (inflorescência + haste) ao final do período reprodutivo. $\mathrm{O}$ acúmulo foi determinado através do produto do teor de nutrientes pela fitomassa seca das mesmas e o acúmulo total, pela soma do acúmulo nas folhas e flores (inflorescência + haste). O silicato de potássio promoveu maior teor e maior acúmulo de nutrientes nas folhas sem uma dose distintiva. A ordem decrescente para a absorção de nutrientes das plantas de gérbera foi de $\mathrm{K}>\mathrm{N}>\mathrm{Ca}>\mathrm{Mg}>\mathrm{P}>\mathrm{S}$ para cloreto e silicato de potássio, ao final do período vegetativo e reprodutivo para as folhas e acúmulo total. $\mathrm{Na}$ inflorescência a ordem de absorção foi de: $\mathrm{K}>\mathrm{N}>\mathrm{P}>\mathrm{Ca}>\mathrm{S}>\mathrm{Mg}$ para as duas fontes utilizadas.

Palavras-chave: Gerbera jamesonii, nutrição de plantas, absorção, nutrientes.

\begin{abstract}
Accumulation of nutrients in pot gerbera according to sources and doses of potassium

Gerbera is one of the several cultivated species of economic importance to agriculture. This is a plant with good durability, resistance to transport and high appreciation by consumers. However, despite the expansion of commercial production of flowers and ornamental plants, research has not grown at the same rate, especially with regard to nutrition and fertilization. The content and accumulation of macronutrients were evaluated in plants of gerbera in two growth stages (vegetative and reproductive), using different concentrations of potassium in the nutrient solution and two sources (chloride and silicate). The experiment was carried out in a greenhouse of the Universidade Estadual Paulista, Botucatu, Brazil. The experimental design was of randomized blocks, with a $2 \times 5$ factorial scheme, comprising two sources of potassium (silicate and chloride) with five concentrations $\left(0,50,100,150\right.$ and $200 \mathrm{mg} \mathrm{L}^{-1}$ of potassium, in nutrient solution), four replications and five plants per plot. The gerbera cultivar used was Red, with dark center, for growing in vase. The levels of macronutrients $(\mathrm{N}, \mathrm{P}, \mathrm{K}, \mathrm{Ca}, \mathrm{Mg}$ and $\mathrm{S})$ were accessed on leaves during the vegetative period and leaves and flowers (inflorescence + stem) on the end of the reproductive period. The accumulation was obtained through the multiplication of the nutrient content with the dry weight of the same ones and the total accumulation was obtained by the sum of accumulation on leaves and flowers (inflorescence + stem). Potassium silicate promoted greater content and accumulation of nutrients in leaves without a distinctive dose. The descending order for the absorption of nutrients of the gerbera plants was $\mathrm{K}>\mathrm{N}>\mathrm{Ca}>\mathrm{Mg}>\mathrm{P}>\mathrm{S}$ for chloride and potassium silicate on the end of the vegetative and reproductive period for leaves and total accumulation. In the reproductive period the order of absorption was $\mathrm{K}>\mathrm{N}>\mathrm{P}>\mathrm{Ca}>\mathrm{S}>\mathrm{Mg}$ for the both sources used.
\end{abstract}

Keywords: Gerbera jamesonii, plant nutrition, absorption, nutrient.

\section{(Recebido para publicação em 7 de dezembro de 2010; aceito em 9 de março de 2012) (Received on December 7, 2010; accepted on March 9, 2012)}

\begin{abstract}
$\mathrm{N}^{\infty}$ os últimos anos o cultivo comercial de gérbera de vaso e de corte (Gerbera jamesonii) tornou-se de grande importância econômica para a floricultura. Ludwig et al. (2010) relatam que o seu cultivo restringia-se à flor de corte, ou seja, diretamente em solo, sendo recente seu comércio como flor envasada. De acordo com Ciranda de cores (2002), isso só se tornou pos-
\end{abstract}

sível com a importação de sementes que resultavam em plantas mais compactas, apropriadas para serem cultivadas em vasos, floreiras e até mesmo nos jardins. Em vaso, esta planta dura entre duas e seis semanas, dependendo da cultivar e dos tratos culturais que receber.

Apesar da expansão da produção comercial de flores e plantas ornamentais no Brasil em geral, a pesquisa não tem acompanhado esse ritmo de crescimento, sendo escassas as informações sobre a produção de gérbera de vaso, principalmente no que diz respeito à nutrição e a adubação. De acordo com Malavolta et al. (1997), estes dois parâmetros estão entre os essenciais para promover a qualidade e proporcionar retornos adequados às culturas agrícolas de importância econômica. 
Estudos sobre a absorção de nutrientes através da análise mineral em determinados estádios fenológicos e órgão amostrados, colaboram para eficiência da nutrição adequada das espécies, predizendo desta forma os nutrientes mais absorvidos e também a época de maior exigência nutricional. De acordo com Camargo et al. (2004), isso implica em menores perdas de adubo e riscos de toxicidade provocados por concentrações salinas exageradas.

O potássio é o elemento mineral mais absorvido pela gérbera, sendo mais requerido até mesmo que o nitrogênio, merecendo dessa forma atenção especial em seu fornecimento, principalmente no estádio de florescimento (Mercurio, 2002; Ludwig et al., 2008). Este nutriente é exportado da folha para o floema com a sacarose, tendo grande importância na geração de gradiente osmótico entre a fonte e o dreno. Assim sendo, a flor como um forte competidor, levará à deficiência de potássio, o que irá limitar o transporte de sacarose para este local, comprometendo a floração (Barbosa et al., 2009).

Ludwig et al. (2008), em pesquisa realizada com quatro cultivares de gérbera e duas soluções nutritivas (50 e $100 \%$ ), avaliando a absorção de nutrientes em duas épocas (período vegetativo e reprodutivo), relataram que a demanda pelos nutrientes foi diferenciada entre as cultivares e a concentração da solução apresentou um efeito marcante no teor e acúmulo de N, P, Ca, Mg e S na planta. Os maiores acúmulos foram registrados no terço final do ciclo de produção, apresentando ordem decrescente de absorção: $\mathrm{K}>\mathrm{N}>\mathrm{Ca}>\mathrm{Mg}>\mathrm{P}>\mathrm{S}$ (respectivamente, $415,327,110,33,32$ e 20 mg planta $\left.^{-1}\right)$.

O objetivo desse estudo foi avaliar o teor e acúmulo de macronutrientes $(\mathrm{N}, \mathrm{P}$, $\mathrm{K}, \mathrm{Ca}, \mathrm{Mg}$ e $\mathrm{S}$ ) em plantas de gérbera, em dois estádios de desenvolvimento (vegetativo e reprodutivo), utilizando-se duas fontes de potássio e diferentes concentrações na solução nutritiva.

\section{MATERIAL E MÉTODOS}

O experimento foi conduzido em casa de vegetação da Universidade
Estadual Paulista, Botucatu (SP), de setembro a novembro de 2008. A média de temperatura e a umidade relativa média do ar no interior da casa de vegetação foram de $23^{\circ} \mathrm{C}$ e $60 \%$, respectivamente, aferidas com o uso de termohigrômetro com sistema automático de coleta e armazenamento de dados.

A casa de vegetação possui uma área útil total de $168 \mathrm{~m}^{2}$ e 2,6 m de pé direito, com estrutura em arco, plástico transparente, laterais de tela branca, cortinas laterais externas, piso pavimentado com concreto e na parte superior possui malha aluminizada termorrefletora com $50 \%$ de sombreamento.

$\mathrm{O}$ delineamento experimental adotado foi em blocos casualizados, empregando o esquema fatorial $2 \times 5$, sendo duas fontes de potássio (silicato e cloreto), cinco concentrações $(0,50$, 100,150 e $200 \mathrm{mg} \mathrm{L}^{-1}$ ) e quatro repetições. As parcelas foram constituídas por cinco plantas por parcela, num total de 200 plantas. O espaçamento adotado foi de 20x20 cm entre os vasos, dispostos em bancadas de 1,2 $\mathrm{m}$ de largura, 11,0 $\mathrm{m}$ de comprimento e $0,8 \mathrm{~m}$ de altura, totalizando uma área útil de 13,2 $\mathrm{m}^{2}$.

As concentrações foram definidas a partir da solução nutritiva utilizada pela empresa Steltenpool. A composição da solução nutritiva base para a cultura, em mg L-1 foi: $217,1 \mathrm{~N}, 53,9 \mathrm{P}, 64 \mathrm{Ca}$, $40 \mathrm{Mg}, 54,4 \mathrm{~S}, 0,2 \mathrm{~B}, 0,08 \mathrm{Cu}, 2,7 \mathrm{Fe}$, 0,2 Mn, 0,04 Mo e 0,08 Zn. Para os tratamentos com cloreto foi utilizada uma fonte com $52,4 \%$ de potássio e $46 \%$ de cloro e para o silicato, utilizou-se um produto comercial com $173 \mathrm{~g} \mathrm{~L}^{-1} \mathrm{de}$ potássio e $171 \mathrm{~g} \mathrm{~L}^{-1}$ de silício.

Para o cultivo das plantas de gérbera foi utilizado vaso $n^{\circ} 14$ com capacidade para um litro com as seguintes dimensões: $11,5 \mathrm{~cm}$ de altura, 12,7 cm (diâmetro maior) e 9,0 cm (diâmetro menor), preenchido com o substrato composto de 20\% terra (Latossolo Vermelho Escuro), $30 \%$ casca de pinus, $30 \%$ comercial 1 (40\% casca de pinus, 30\% vermiculita, $30 \%$ casca de arroz carbonizada) e $20 \%$ comercial 2 (75\% casca de pinus e $25 \%$ de acícula de pinus) através da densidade úmida. As características químicas do substrato de acordo com metodologia de Raij et al. (2001) Foram: $\mathrm{pH}_{(\mathrm{CaCl} 2)}=4,9$; $\mathrm{P}_{\text {(resina) }}=246 \mathrm{mg} \mathrm{dm}^{-3}, \mathrm{~K}=8, \mathrm{Ca}=3, \mathrm{Mg}$
$=59, \mathrm{SB}=144 \mathrm{mmol}_{\mathrm{c}} \mathrm{dm}^{3}$, respectivamente; $\mathrm{CTC}=205, \mathrm{~V} \%=70, \mathrm{Cu}=3$, $\mathrm{Fe}=209, \mathrm{Mn}=16 \mathrm{e} \mathrm{Zn}=6,3 \mathrm{mg} \mathrm{dm}^{-3}$, respectivamente.

Utilizou-se a cultivar de gérbera Red, que tem como característica principal o centro escuro, proveniente da geração $\mathrm{F}_{1}$ do grupo Festival, da empresa Sakata Seeds Sudamerica ${ }^{\circledR}$, pertencentes à Série Dark Eyes, para cultivo em vaso. As mudas foram adquiridas de empresa especializada permanecendo nesse estádio por aproximadamente um mês. Quando estas apresentavam quatro folhas definitivas, procedeu-se o transplante colocando-se uma muda por vaso. Após o transplante as mudas ficaram em fase de aclimatação aproximadamente por um mês, ou seja, ficaram todas juntas sem espaçamento entre vasos, sob malha aluminizada termorrefletora que mantinha a intensidade luminosa de aproximadamente 25.000 Lux. Após o período de aclimatação, a necessidade de intensidade luminosa para a planta aumenta, sendo mantida na faixa de 40.000 a 45.000 Lux, com o manejo da abertura e fechamento da malha aluminizada termorrefletora. Essas leituras foram realizadas com uso de um luxímetro digital portátil.

A fertirrigação foi realizada manualmente, aplicando-se o método da pesagem, onde um vaso por parcela era pesado diariamente no período da manhã. No estádio vegetativo as plantas foram mantidas na faixa de $40-20 \%$ da água disponível no substrato e no reprodutivo na faixa de $50-30 \%$.

Para a obtenção da capacidade de recipiente, ou seja, volume máximo de água retido pelo substrato no recipiente, um vaso por parcela de cada tratamento foi saturado e também determinado o seu peso seco. O procedimento da saturação consiste em deixar o vaso em recipiente preenchido com água, até a altura mediana do vaso, por 24 horas. Após a saturação, os recipientes foram retirados e deixou-se o vaso por mais 24 horas para escoamento da água gravitacional. Decorridas 24 horas, os vasos novamente foram pesados, e desse valor era subtraído o peso seco obtendo-se assim a máxima quantidade de água disponível no substrato. Com 
o valor encontrado foi estipulado o intervalo da quantidade de fertirrigação a ser aplicada.

As soluções contendo os tratamentos foram aplicadas diariamente com a solução nutritiva utilizada para o cultivo de gérbera. Para isso, adicionava-se sempre a metade da fertirrigação exigida com as soluções dos tratamentos e a outra metade via solução nutritiva.

As determinações do teor e acúmulo de macronutrientes foram realizadas ao final do estádio vegetativo e outra no final do estádio reprodutivo, em uma planta por parcela, totalizando quatro plantas por tratamento, sendo que no período reprodutivo as plantas foram divididas em folhas e flores (inflorescência + haste). Estas foram lavadas em água corrente e em água com detergente, passaram em seguida por duplo enxágue em água deionizada. Logo após, foram acondicionadas em sacos de papel Kraft, identificadas com os respectivos tratamentos e postas para secar em estufa de ventilação forçada mantida a aproximadamente $60^{\circ} \mathrm{C}$ por 48 horas, até atingir massa constante. Após a avaliação da fitomassa seca da parte aérea das duas coletas, o material foi moído em moinho tipo "Willey". Esse material foi encaminhado para análise química de macronutrientes, seguindo metodologia recomendada por Malavolta et al. (1997). O acúmulo foi calculado através do produto da fitomassa seca e o teor de nutrientes.

Os resultados dos tratamentos foram submetidos à análise de variância pelo teste F. Os resultados obtidos para as duas fontes utilizadas (cloreto e silicato de potássio) foram submetidos ao teste Tukey a 5\% de probabilidade e análise de regressão para as doses, na qual foram testados os modelos linear e quadrático. Quando houve interação entre as fontes e as doses, foi realizado desdobramento e para as doses adotou- -se a equação de regressão que melhor se ajustou aos dados, a qual foi escolhida com base na significância dos coeficientes de regressão a $1 \%(* *)$ e $5 \%\left(^{*}\right)$ de probabilidade pelo teste $\mathrm{F}$ e no maior valor dos coeficientes de determinação $\left(\mathrm{R}^{2}\right)$.

\section{RESULTADOS E DISCUSSÃO}

As fontes de potássio utilizadas apresentaram resposta diferenciada nas folhas, no período vegetativo, para teor e acúmulo de N. O silicato de potássio foi mais eficiente, sendo encontrados maiores valores de teor e acúmulo de $\mathrm{N}$ nas folhas, 31,1 $\mathrm{g} \mathrm{kg}^{-1}$ e 150,9 mg planta $^{-1}$, respectivamente (Tabela 1). Observou-se entre as doses, ajuste linear negativo para o teor de $\mathrm{N}$ na folha no período reprodutivo (Figura $1 \mathrm{~A})$. $\mathrm{Na}$ flor, ocorreu interação significativa entre fonte e dose para teor de $\mathrm{N}$, com ajuste linear negativo para cloreto e ajuste

Tabela 1. Teor e acúmulo de macronutrientes nas folhas e flores de gérbera (inflorescência + haste) ao final do período vegetativo (42 DAA) e reprodutivo (60 DAA) e acúmulo total (60 DAA) [content and accumulation of nutrients in leaves and flowers of gerbera (inflorescence + stem) at the end of the growing season (42 DAA) and reproductive stage (60 DAA) and accumulation total at 60 DAA]. Botucatu, UNESP, 2008.

\begin{tabular}{|c|c|c|c|c|c|c|c|c|c|c|c|c|}
\hline \multirow{4}{*}{ Fonte } & \multicolumn{12}{|c|}{ Nutrientes } \\
\hline & $\mathbf{N}$ & $\mathbf{P}$ & $\mathbf{K}$ & $\mathbf{C a}$ & Mg & $\mathbf{S}$ & $\mathbf{N}$ & $\mathbf{P}$ & $\mathbf{K}$ & $\mathbf{C a}$ & $\mathbf{M g}$ & $\mathbf{S}$ \\
\hline & \multicolumn{6}{|c|}{$\left(\mathrm{g} \mathrm{kg}^{-1}\right)$} & \multicolumn{6}{|c|}{$\left(\mathrm{mg} \mathrm{planta}^{-1}\right)$} \\
\hline & \multicolumn{12}{|c|}{ Folha (42DAA) } \\
\hline Cloreto & $29,8 b$ & $2,5 b$ & $37,7 \mathrm{a}$ & $11,1 \mathrm{a}$ & $4,1 \mathrm{a}$ & $2,1 \mathrm{a}$ & $135,2 b$ & $11,6 \mathrm{~b}$ & $171,3 \mathrm{a}$ & $50,3 a$ & $18,8 \mathrm{~b}$ & $9,5 b$ \\
\hline Silicato & $31,1 \mathrm{a}$ & $2,7 \mathrm{a}$ & $39,9 \mathrm{a}$ & $11,2 \mathrm{a}$ & $4,3 \mathrm{a}$ & $2,2 \mathrm{a}$ & $150,9 \mathrm{a}$ & $13,3 \mathrm{a}$ & $194,1 \mathrm{a}$ & $54,0 \mathrm{a}$ & $20,9 \mathrm{a}$ & $10,6 \mathrm{a}$ \\
\hline \multirow[t]{2}{*}{$\mathrm{CV}(\%)$} & 5,9 & 8,2 & 9,9 & 12,3 & 11,0 & 10,2 & 15,3 & 13,8 & 20,7 & 16,3 & 15,5 & 14,2 \\
\hline & \multicolumn{12}{|c|}{ Folha (60DAA) } \\
\hline Cloreto & $27,9 \mathrm{a}$ & $2,4 \mathrm{~b}$ & $37,9 \mathrm{a}$ & $14,4 \mathrm{a}$ & $4,6 \mathrm{a}$ & $1,7 \mathrm{~b}$ & $172,3 \mathrm{a}$ & $15,0 \mathrm{~b}$ & $230,6 a$ & $87,6 a$ & $28,7 \mathrm{a}$ & $10,6 \mathrm{~b}$ \\
\hline Silicato & $28,5 \mathrm{a}$ & $2,6 a$ & $37,3 \mathrm{a}$ & $13,2 \mathrm{a}$ & $4,3 \mathrm{~b}$ & $1,8 \mathrm{a}$ & $189,9 a$ & $17,4 \mathrm{a}$ & $249,5 \mathrm{a}$ & $88,3 \mathrm{a}$ & $28,7 \mathrm{a}$ & $12,1 \mathrm{a}$ \\
\hline \multirow[t]{2}{*}{$\mathrm{CV}(\%)$} & 5,8 & 8,2 & 9,9 & 13,5 & 10,5 & 6,8 & 15,6 & 17,3 & 17,9 & 17,6 & 14,8 & 15,6 \\
\hline & \multicolumn{12}{|c|}{ Flor (60DAA) } \\
\hline Cloreto & $27,1 \mathrm{a}$ & $3,3 a$ & $29,6 \mathrm{a}$ & $3,4 \mathrm{a}$ & $1,68 \mathrm{a}$ & $2,1 \mathrm{a}$ & $115,6 \mathrm{a}$ & $14,0 \mathrm{~b}$ & $124,7 \mathrm{a}$ & $13,9 \mathrm{a}$ & $7,1 \mathrm{a}$ & $9,1 \mathrm{a}$ \\
\hline Silicato & $26,4 \mathrm{a}$ & $3,5 \mathrm{a}$ & $29,1 \mathrm{a}$ & $3,5 \mathrm{a}$ & $1,70 \mathrm{a}$ & $2,1 \mathrm{a}$ & $120,2 \mathrm{a}$ & $15,8 \mathrm{a}$ & $132,0 \mathrm{a}$ & $15,4 \mathrm{a}$ & $7,9 \mathrm{a}$ & $9,8 \mathrm{a}$ \\
\hline \multirow[t]{4}{*}{$\mathrm{CV}(\%)$} & 6,0 & 8,7 & 9,6 & 22,6 & 10,1 & 8,5 & 22,0 & 18,6 & 21,9 & 25,7 & 22,5 & 19,8 \\
\hline & \multicolumn{12}{|c|}{ Acúmulo total (60DAA) } \\
\hline & \multicolumn{2}{|c|}{$\mathbf{N}$} & \multicolumn{2}{|c|}{$\mathbf{P}$} & \multicolumn{2}{|c|}{$\mathbf{K}$} & & $\mathbf{C a}$ & \multicolumn{2}{|c|}{ Mg } & \multicolumn{2}{|c|}{$\mathbf{S}$} \\
\hline & \multicolumn{12}{|c|}{$\left(\mathrm{mg} \mathrm{planta}^{-1}\right)$} \\
\hline Cloreto & \multicolumn{2}{|c|}{$288,0 \mathrm{a}$} & \multicolumn{2}{|c|}{$29,0 \mathrm{~b}$} & \multicolumn{2}{|c|}{$355,4 a$} & \multicolumn{2}{|c|}{$101,6 a$} & \multicolumn{2}{|c|}{$35,8 \mathrm{a}$} & \multicolumn{2}{|c|}{$19,8 b$} \\
\hline Silicato & \multicolumn{2}{|c|}{$310,0 \mathrm{a}$} & \multicolumn{2}{|c|}{$33,3 \mathrm{a}$} & \multicolumn{2}{|c|}{$381,6 a$} & \multicolumn{2}{|c|}{$103,7 \mathrm{a}$} & \multicolumn{2}{|c|}{$36,7 \mathrm{a}$} & \multicolumn{2}{|c|}{$22,0 \mathrm{a}$} \\
\hline $\mathrm{CV}(\%)$ & \multicolumn{2}{|c|}{12,5} & \multicolumn{2}{|c|}{11,5} & \multicolumn{2}{|c|}{12,7} & & & & & & \\
\hline
\end{tabular}

Valores seguidos pela mesma letra na coluna não diferem entre si pelo teste de Tukey a 5\%; DAA= dias após a aclimatação (values followed by the same letter in column do not differ by Tukey test at 5\%; DDA= days after acclimation). 

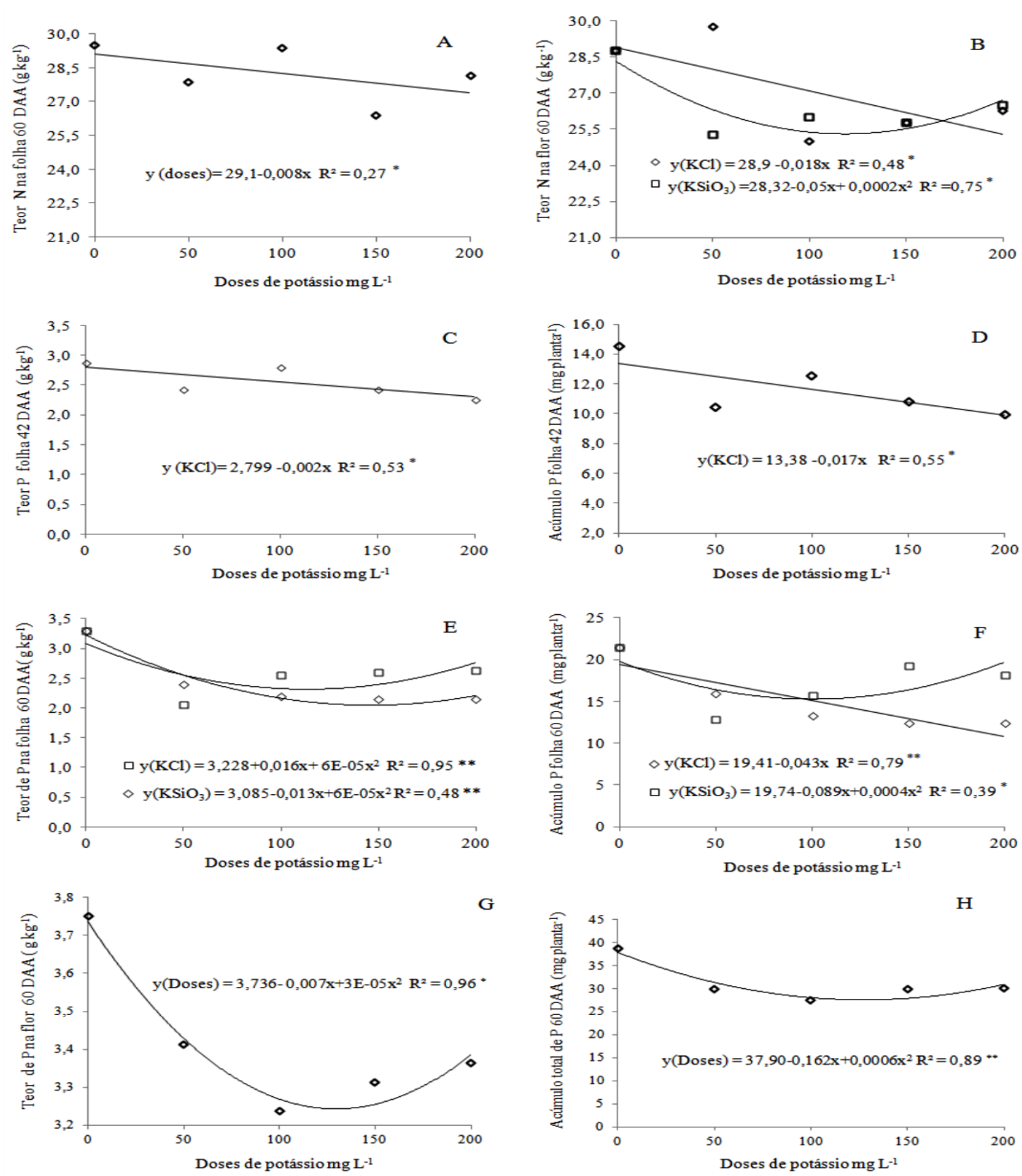

Figura 1. Doses de potássio na solução nutritiva utilizando-se de duas fontes, cloreto e silicato de potássio em plantas de gérbera aos 42 e 60 dias após aclimatação (DAA) para: (A) teor de $\mathrm{N}$ na folha, (B) teor de $\mathrm{N}$ na flor, (C) teor de $\mathrm{P}$ na folha, (D) acúmulo de P na folha, (E) teor de $\mathrm{P}$ na folha, $(\mathrm{F})$ acúmulo de $\mathrm{P}$ na folha, $(\mathrm{G})$ teor de $\mathrm{P}$ na flor e $(\mathrm{H})$ acúmulo total de $\mathrm{P}$ [doses of potassium in the nutrient solution using two sources, chloride and potassium silicate in plants of gerbera at 42 and 60 days after the acclimation (DAA), being: (A) N content in leaf, (B) N content in flower, (C) P content in leaf, (D) accumulation of P in leaf, (E) P content in leaf, (F) accumulation of P in leaf, (G) $\mathrm{P}$ content in leaf and $(\mathrm{H})$ accumulation of total P]. Botucatu, UNESP, 2008.

quadrático para o silicato (Figura 1B). Nesta, a maior diferença entre fontes ocorreu para a dose de $50 \mathrm{mg} \mathrm{L}^{-1} \mathrm{de}$ potássio, com $29,7 \mathrm{~g} \mathrm{~kg}^{-1}$, para o cloreto e $25 \mathrm{~g} \mathrm{~kg}^{-1}$ para o silicato.

$\mathrm{O}$ teor de $\mathrm{N}$ na folha, de maneira geral, foi $7,4 \%$ maior aos 42 DAA em relação aos 60 DAA (Tabela 1), compro- vando a importância deste nutriente no desenvolvimento vegetativo da planta. Fazendo um comparativo da absorção de $\mathrm{N}$ entre as folhas e as flores aos 60 DAA, verificou-se que estes foram semelhantes, não mostrando diferença significativa no requerimento deste nutriente. Oldoni (2009) relata que o nitrogênio é um nutriente importante na fase de crescimento e também na fase reprodutiva da gérbera, contribuindo principalmente para o crescimento da haste floral.

Ludwig et al. (2008), em trabalho realizado com quatro cultivares de gérbera e solução nutritiva de 50 e $100 \%$, 

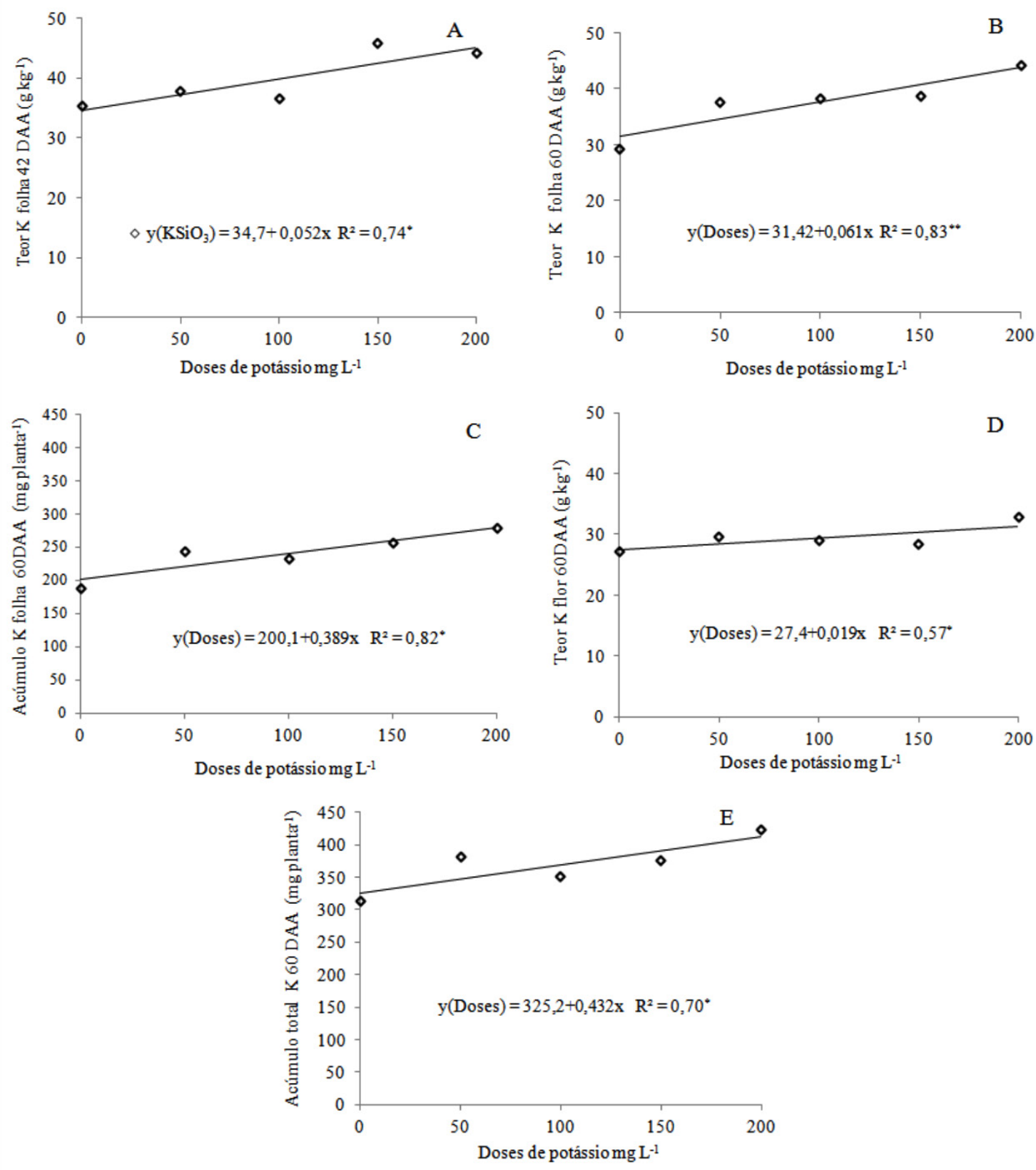

Figura 2. Doses de potássio utilizando-se duas fontes, cloreto e silicato de potássio em plantas de gérbera aos 42 e 60 dias após aclimatação (DAA) para: (A) teor de K na folha aos $42 \mathrm{DAA}$, (B) teor de $\mathrm{K}$ na folha aos $60 \mathrm{DAA},(\mathrm{C})$ acúmulo de K na folha, (D) teor de K na flor e (E) acúmulo total de $\mathrm{K}$ [doses of potassium using two sources, chloride and potassium silicate in plants of gerbera at 42 and 60 days after the acclimation (DAA), being: (A) K content in leaf 42 DAA, (B) K content in leaf 60 DAA, (C) accumulation of K in leaf, (D) K content in flower and (E) total accumulation of K]. Botucatu, UNESP, 2008.

relatam que houve a duplicação da absorção total de $\mathrm{N}$ ao final do ciclo de cultivo, corroborando com os resultados obtidos neste estudo, visto que até os 42 DAA, praticamente metade do $\mathrm{N}$ havia sido incorporado à planta (48\%) e que num prazo de 18 dias houve a duplicação deste (Tabela 1).

Para o P, foi observado resultado significativo na interação entre fontes e doses, para teor e acúmulo nas folhas no período vegetativo e reprodutivo. No período vegetativo houve ajuste linear negativo para teor e acúmulo, somente para o cloreto (Figuras 1C e D). Já no período reprodutivo o melhor ajuste na regressão para o cloreto foi quadrático para o teor e linear negativo para o acúmulo. Para o silicato, tanto para o teor quanto para o acúmulo, a equação que melhor se ajustou aos dados foi a quadrática (Figura $1 \mathrm{E}$ e F).

Entre as doses de potássio houve diferença significativa para teor na flor aos 60 DAA e acúmulo total, com ajuste quadrático nas duas avaliações (Figuras $1 \mathrm{G}$ e $\mathrm{H}$ ). Na flor houve resultado significativo somente entre as fontes para o acúmulo de fósforo, onde o silicato foi mais eficiente em promover resultados 

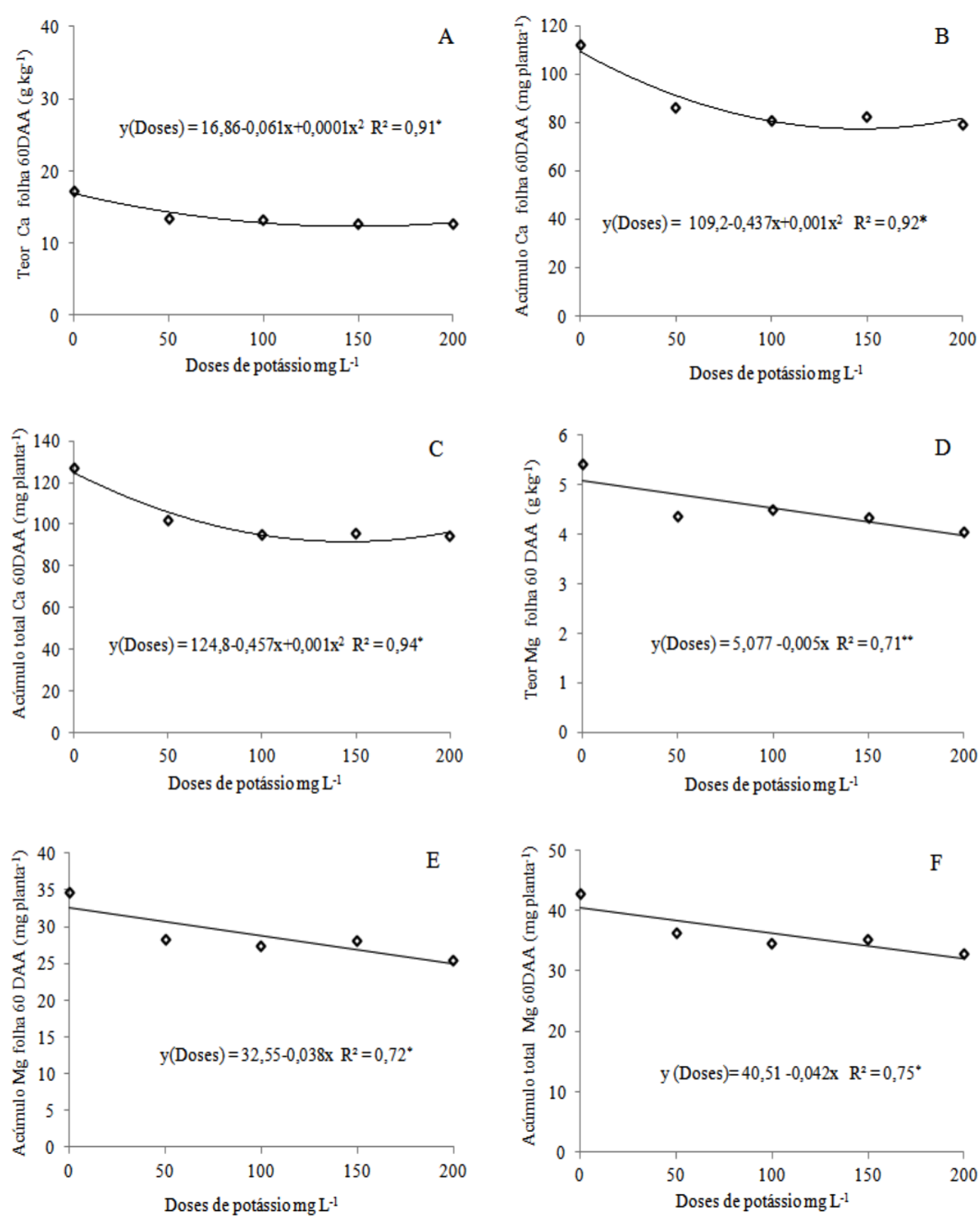

Figura 3. Doses de potássio utilizando-se duas fontes, cloreto e silicato de potássio em plantas de gérbera aos 60 dias após aclimatação (DAA) para: (A) teor de $\mathrm{Ca}$ na folha, (B) acúmulo de Ca na folha, (C) acúmulo total de $\mathrm{Ca}$, (D) teor de $\mathrm{Mg}$ na folha, (E) acúmulo de $\mathrm{Mg}$ na folha e $(\mathrm{F})$ acúmulo total de $\mathrm{Mg}$ [doses of potassium using two sources, chloride and potassium silicate in plants of gerbera at 60 days after the acclimation (DAA), being: (A) Ca content in leaf, (B) accumulation of Ca in leaf, (C) total accumulation of Ca, (D) Mg content in leaf, (E) accumulation of $\mathrm{Mg}$ in leaf and (F) total accumulation of Mg]. Botucatu, UNESP, 2008.

superiores deste nutriente (Tabela 1).

A quantidade de $\mathrm{P}$ acumulada em plantas de gérbera ocorreu de forma ainda mais intensa que o observado para $\mathrm{N}$, tendo este triplicado nos últimos 18 dias, ou seja, a maior demanda coincide com o terço final do ciclo da gérbera, resultados semelhantes ao observado por Ludwig et al. (2008). Ao contrário do que ocorreu para o $\mathrm{N}$, foi observado maior teor deste nutriente nas flores em relação às folhas. Barbosa et al. (2009) relatam que no início do desenvolvimento das plantas ornamentais as quantidades exigidas de $\mathrm{P}$ são menores, aumentando com o tempo, coincidindo com o florescimento.

Para as fontes, nas duas épocas e tecido da planta avaliada, o silicato de potássio contribuiu de maneira mais efetiva em aumentar o teor e acúmulo de P na parte aérea das plantas de gérbera. Lana et al. (2003) descrevem que o silício atua diminuindo o efeito tóxico 
do $\mathrm{Mn}, \mathrm{Fe}$ e outros metais pesados e aumenta a absorção e metabolismo de elementos, tais como o $\mathrm{P}$, como pode ser observado neste trabalho.

Para o K houve efeito significativo para interação entre fonte e dose apenas para teor aos 42 DAA, com ajuste de regressão linear positiva, somente para o silicato (Figura 2 A). Para as doses houve ajuste de regressão linear positiva no período reprodutivo para teor e acúmulo na folha, teor na inflorescência e acúmulo total (Figuras 2 B, C, D e E). O teor de $\mathrm{K}$ na folha foi semelhante nos dois períodos avaliados, porém o acúmulo deste nutriente foi superior no período reprodutivo (Tabela 1 ).

Mercurio (2002) propõe como teor adequado de $\mathrm{K}$ para a gérbera valores entre 30,6 a $36,4 \mathrm{~g} \mathrm{~kg}^{-1}$. Os teores de $\mathrm{K}$ na folha ao final do período vegetativo e reprodutivo estão acima do proposto por este autor. Ludwig et al. (2008) relatam teores de $\mathrm{K}$ em folhas de gérbera também elevados, sem no entanto observarem sintomas de excesso, o que também não foi observado nesse estudo.

Para o Ca houve efeito estatístico significativo apenas entre as doses de potássio com ajuste quadrático para teor e acúmulo nas folhas e acúmulo total (Figuras 3A, B e C). O teor de $\mathrm{Ca}$ nas folhas foi inferior no estádio vegetativo em relação ao reprodutivo e, o teor na flor inferior a estes dois (Tabela 1), o que pode ser explicado devido à baixa ou nula mobilidade do cálcio no floema (Pedrosa et al., 2000). Diferente disso, Ludwig et al. (2008) encontraram proporcionalidade nos dois períodos analisados, indicando que este nutriente deve ser fornecido durante todo o ciclo.

$\mathrm{O}$ teor de $\mathrm{Ca}$ obtido neste estudo está abaixo do limite crítico proposto por Mercurio (2002), de 16,6 $\mathrm{g} \mathrm{kg}^{-1}$. Entretanto Ludwig et al. (2008), encontraram valores entre 7 e $12 \mathrm{~g} \mathrm{~kg}^{-1}$, ou seja, semelhantes aos encontrados no presente estudo. Apesar dos valores estarem abaixo do recomendado não foi verificado sintoma de deficiência.

Para o Mg houve diferença significativa entre as fontes para acúmulo nas folhas aos 42 DAA, com maiores valores $\left(20,9 \mathrm{mg}\right.$ planta $\left.^{-1}\right)$ para o silicato. Em relação ao teor, o maior valor $\left(4,6 \mathrm{~g} \mathrm{~kg}^{-1}\right)$ foi para cloreto aos 60 DAA
(Tabela 1). Em relação às doses, houve resultado significativo para o teor e acúmulo nas folhas e total no período reprodutivo com ajuste linear negativo (Figuras 3D, E e F).

$\mathrm{O}$ teor e acúmulo de $\mathrm{Mg}$ nas folhas no estádio reprodutivo foi superior ao encontrado no vegetativo. Nas flores, tanto o teor quanto o acúmulo foram inferiores em relação às folhas (Tabela 1). Ludwig et al. (2008) encontraram teor inferior ao final do período vegetativo e reprodutivo com valores para solução nutritiva de $50 \%$, entre 2,9 e $2,7 \mathrm{~g} \mathrm{~kg}^{-1}$ e solução nutritiva de $100 \%$ entre 3,3 e $3,1 \mathrm{~g} \mathrm{~kg}^{-1}$, respectivamente.

O K ficou acima do limite superior proposto para a gérbera, o $\mathrm{Ca}$ e $\mathrm{Mg}$ abaixo do considerado adequado, mostrando que a relação $\mathrm{K} / \mathrm{Ca}$ e $\mathrm{K}$ / $\mathrm{Mg}$ não estava balanceada, refletindo em menor absorção de $\mathrm{Ca}$ e $\mathrm{Mg}$. Isso pode ser observado ao analisar o coeficiente de correlação negativo entre $\mathrm{K}$ e Ca (r: $-0,75^{*}, \mathrm{r}:-0,64^{*}$ e r: $-0,62^{*}$, para teor e acúmulo na folha aos 60 DAA e acúmulo total, respectivamente). Essa mesma tendência pode ser observada para a relação entre $\mathrm{K}$ e $\mathrm{Mg}\left(\mathrm{r}:-0,83^{* *}\right.$, r: $-0,69^{* *}$ e r: $-0,66^{*}$, para teor e acúmulo na folha aos 60 DAA e acúmulo total). No entanto entre $\mathrm{Ca}$ e $\mathrm{Mg}$ a correlação foi positiva (r: $0,93^{* *}, \mathrm{r}: 0,96^{* *}, \mathrm{r}: 0,88^{* *}$ e r: $0,97^{* *}$, para acúmulo na folha aos 42 DAA e acúmulo na folha, flor e total aos 60 DAA, respectivamente). Resultados semelhantes foram observados por Casarini (2004) e Ludwig et al. (2008).

A absorção de Ca pelas raízes é fortemente influenciada pela concentração de $\mathrm{K}$ na zona radicular, devido a este antagonismo (Casarini, 2004). Em baixas concentrações o Ca exerce um efeito estimulante na absorção de potássio, ao passo que em altas concentrações ocorre redução na absorção de potássio pelas plantas (Assis, 1995), como pode ser observado nos resultados encontrados.

No período vegetativo, o silicato de potássio proporcionou maior acúmulo de S na folha em relação ao cloreto, 10,6 $\mathrm{mg} \mathrm{planta}^{-1}$, o que se refletiu também no teor e acúmulo de $\mathrm{S}$ na folha no período reprodutivo, com $1,8 \mathrm{~g} \mathrm{~kg}^{-1}$ e $12,1 \mathrm{mg}$ planta $^{-1}$, respectivamente (Tabela 1 ). Kamenidou et al. (2010), em trabalho realizado com gérbera de corte, utilizan- do fontes e doses de silício, encontraram resultados superiores, com teores na folha entre 2,6 a 3,1 $\mathrm{g} \mathrm{kg}^{-1}$ e observaram que as fontes de silício colaboraram para o aumento do teor deste nutriente independente da dose. No entanto, Camargo et al. (2008), encontraram teores semelhantes de $\mathrm{S}\left(2,3 \mathrm{~g} \mathrm{~kg}^{-1}\right)$ no cultivo de Aster ericoides, planta ornamental da mesma família da gérbera. O teor de $\mathrm{S}$ na folha não variou durante todo o ciclo de cultivo, sendo que na flor o teor foi menor. $\mathrm{O}$ acúmulo deste nutriente praticamente dobrou ao final do ciclo de cultivo.

O silicato de potássio promoveu maior teor e acúmulo de nutrientes nas folhas sem uma dose distintiva. A ordem decrescente para a absorção de nutrientes das plantas de gérbera foi a mesma para cloreto e silicato de potássio: $\mathrm{K}>\mathrm{N}>\mathrm{Ca}>\mathrm{Mg}>\mathrm{P}>\mathrm{S}$, ao final do período vegetativo e reprodutivo para a folha e acúmulo total. Na flor a ordem de absorção foi $\mathrm{K}>\mathrm{N}>\mathrm{P}>\mathrm{Ca}>\mathrm{S}>\mathrm{Mg}$ para as duas fontes utilizadas.

\section{AGRADECIMENTOS}

À Capes e CNPq pelas bolsas concedidas aos autores. A empresa Sakata e Steltenpool pelo material concedido no auxílio desta pesquisa.

\section{REFERÊNCIAS}

ASSIS RP. 1995. Nutrição mineral e crescimento de mudas de dendezeiro (Elaeis guinensis jacq.) em função de diferentes relações entre K, Ca e Mg na solução nutritiva. 1995. Lavras: UFLA. 41p. (Tese mestrado).

BARBOSA JG; BARBOSA MS; MUNIZ MA; GROSSI JA. 2009. Nutrição mineral e adubação de plantas ornamentais. Informe Agropecuário 30: 16-21.

CAMARGO MS; SHIMIZU LK; SAITO MA; KAMEOKA CH; MELLO SC; CARMELLO QAC. 2004. Crescimento e absorção de nutrientes pelo Lisianthus (Eustoma grandiflorum) cultivado em solo. Horticultura Brasileira 22: 143-146.

CAMARGO MS; MELLO SC; CARMELLO QAC. 2008. Adubação nitrogenada e potássica do Aster ericoides cultivado em solo sob estufa. Horticultura Brasileira 26: 190-193.

CASARINI E. 2004. Doses de N e K aplicados via fertirrigação na cultura da roseira (Rosa sp.) em ambiente protegido. Piracicaba: USPESALQ. 120 p (Tese doutorado).

Ciranda de cores. 2002. Globo Rural 203. Disponível em http://revistagloborural.globo. 
com/EditoraGlobo/componentes/article/ edg_article_print/1,3916,378033-1641-1,00. html. Acessado em 20 de setembro de 2010.

KAMENIDOU S; CAVINS TJ; MAREK S. 2010. Silicon supplements affect floricultural quality traits and elemental nutrient concentrations of greenhouse produced gerbera. Scientia Horticulturae 123: 390-394.

LANA RMQ; KORNDÖRFER GH; ZANÃO JUNIOR LA; SILVA AF; LANA AMQ. 2003. Efeito do silicato de cálcio sobre a produtividade e acumulação de silício no tomateiro. Bioscience Journal 19: 15-20.

LUDWIG F; FERNANDES DM; MOTA PRD;
VILLAS BÔAS RL. 2008. Macronutrientes em cultivares de gérbera sob dois níveis de fertirrigação. Horticultura Brasileira 26: 068-073.

LUDWIG F; GUERRERO AC; FERNANDES DM; VILLAS BOAS RL. 2010. Análise de crescimento de gérbera de vaso conduzida em diferentes substratos. Horticultura Brasileira 28: 70-74.

MALAVOLTA E; VITTI GC; OLIVEIRA SA. 1997. Avaliação do estado nutricional das plantas: princípios e aplicações. Piracicaba: Potafos. 319p.

MERCURIO G. 2002. Gerbera cultivation in greenhouse. De Kwakel: Schreurs. 206p.

OLDONI CM. 2009. Produção de gérberas. Informe Agropecuário 30: 67-73.

PEDROSA MW; BARBOSA JG; MARTINEZ HEP; CARDOSO AA. 2000. Concentração e acúmulo de nutrientes em plantas de Gipsophila paniculata L. cultivada em solução nutritiva. Revista Brasileira de Horticultura Ornamental 6: 19-30.

RAIJ B van; ANDRADE JC; CANTARELLA H; QUAGGIO JA. 2001. Análise química da fertilidade dos solos tropicais. Campinas: IAC. 285 p. 